ELECTRONIC PAPER

\title{
The inhibition of mercury absorption by dietary ethanol in humans: cross-sectional and case-control studies
}

\author{
M D Martin, C Naleway
}

Occup Environ Med 2004;61:e8 (http://www.occenvmed.com/cgi/content/full/61/2/e8)

Background: Since the inhibition of mercury absorption by ethanol was serendipitously discovered in 1965,' a limited number of small number studies with both animal and human subjects have reported results consistent with this finding.

Aims: To investigate this phenomenon in a large scale human study with low level $\mathrm{Hg}$ exposed dentists.

See end of article for authors' affiliations

.....................

Correspondence to: Associate Professor M D Martin, Dept of Oral Medicine, Box 356370

University of Washington, Seattle, WA 98195, USA mickeym@

u.washington.edu

Accepted

8 September 2003
Methods: Data were collected for a sample of 1171 dentists, and both cross sectional and case-control methods were utilised to examine the data.

Results: Abstainers $(n=345)$ had significantly higher urinary mercury concentrations $(\mathrm{HgU})$ than drinkers $(\mathrm{n}=826): 5.4 \mu \mathrm{g} / \mathrm{l} v 4.8 \mu \mathrm{g} / \mathrm{l}$. Multiple linear regression showed a significant effect of ethanol dose on $\mathrm{HgU}$ after adjusting for potential confounders. A case-control analysis in which cases were defined as those individuals with urinary $\mathrm{Hg}$ concentrations of $\geqslant 15 \mu \mathrm{g} / \mathrm{l}(\approx$ top $5 \%)$, and controls as those with concentrations of $<1.0 \mu \mathrm{g} / \mathrm{l}(\approx$ bottom $5 \%$ ), showed a clear protective dose-response relation; there was a decreasing risk of being a "case" (having an $\mathrm{HgU} \geqslant 15 \mu \mathrm{g} / \mathrm{l}$ ) with increasing ethanol consumption. The significance of the adjusted model is $p<0.001$, and the $\chi^{2}$ test for trend across ethanol consumption categories in the adjusted model is $p<0.05$, confirming the dose-response relation.

Conclusion: We believe that this straightforward investigation provides the first specific confirmation in a large scale human study of the inhibitory effect of ethanol on urinary mercury concentration, and by inference, on mercury absorption.
$\mathrm{T}$ hat the ingestion of ethanol inhibits the absorption of inhaled mercury vapour in humans was serendipitously discovered by F Nielsen Kudsk during an investigation of the fate of mercury vapour absorbed through the lungs in a human subject experiment reported in 1965. ${ }^{1}$ One of four subjects ingested an unscheduled quantity of beer containing approximately $9 \mathrm{~g}$ of ethanol just prior to an experimental session, and showed a reduced absorption of inhaled mercury vapour during the session. The same investigator reported a small subsequent confirmatory follow up human study $(\mathrm{n}=4)$ in which the ingestion of ethanol was shown to inhibit the absorption of mercury vapour, ${ }^{2}$ and an in vitro study which showed that mercury vapour uptake by human blood was reduced by the addition of $0.2 \%$ ethanol. ${ }^{3}$

Elemental mercury, the form used in dentistry, and which provides both occupational exposures to dentists as well as exposures to patients with mercury amalgam fillings, is rapidly absorbed into the blood after inhalation. Once there, much of it is rapidly oxidised to the mercuric ion through the catalase-hydrogen peroxide pathway. Much of it then binds to circulating proteins or those in red blood cells, and is held in high molecular weight complexes. Hursh and colleagues ${ }^{4}$ conducted mercury vapour inhalation experiments in humans with three ethanol exposed and two ethanol unexposed subjects, also finding that mercury retention was reduced by ethanol ingestion. This study additionally found a reduction in the red blood cell mercury content of ethanol exposed subjects. This same report included animal studies (mouse and rat) in which ethanol pretreated animals showed significantly lower concentrations of mercury in organ tissues following mercury exposure. A dose dependent increase of mercury exhalation in mercury pretreated and subsequently ethanol exposed mice was reported in a 1981 study. ${ }^{5}$ The authors concluded that ionic mercury in tissue could be mobilised via reduction, and that $\mathrm{Hg}^{++}$in tissues was more labile than previously recognised.
A larger scale human study comparing mercury exposed chloralkalai workers $(n=89)$ to controls $(n=75)$ found no significant relation between between self reported alcohol intake and mercury levels in blood or urine. ${ }^{6}$ Alternatively, a study among Faroe Island residents which examined umbilical cord blood and the effect of maternal seafood diet on fetal exposure to lead, mercury, and selenium found a significant difference in cord blood mercury concentration between abstainers and ethanol drinkers, with abstainers having higher concentrations than alcohol users. ${ }^{7}$ This finding was considered by the authors to be epidemiological confirmation of the phenomenon of the previously described inhibition of mercury absorption by ethanol. A recent study of long term low level mercury exposed humans $(n=14)$ found that dosing with ethanol increased the mercury concentration in exhaled air, and that higher doses of ethanol resulted in higher mercury levels in exhaled air as well as a longer time to return to background levels. ${ }^{8}$

Magos et al suggested from animal studies that the mechanism by which ingested ethanol inhibits the absorption of mercury vapour is most probably the inhibition of the oxidation of mercury, and that the site of this inhibition is not necessarily restricted to the blood. ${ }^{9}$ No significant alternative theories have been published to date, although a number of subsequent animal studies have presented the same conclusion.

The purpose of the current study was to examine the relation of self reported ethanol intake to recent mercury exposure as measured by urinary mercury concentration $(\mathrm{HgU})$ among a large sample of low level occupationally exposed dentists.

\section{METHODS}

Subjects were recruited at a large dental professional meeting. Dentists are well known to experience low level mercury exposure through their use of elemental $\mathrm{Hg}$ in preparing and 
placing dental amalgam. Unadjusted spot urine samples, which have been shown to provide reasonable estimates of 24 hour $\mathrm{HgU}$ concentrations for cross sectional studies ${ }^{10} 11$ as well as questionnaire data concerning practice, personal, and office characteristics were collected from 1171 dentists over a three day period. Urinary mercury concentrations were one of several laboratory tests offered to dentists attending a national professional dental meeting. The sample was comprised of all dentists at the meeting who electively chose to have a urinary mercury concentration performed. This sample did not significantly differ from all dentists nationally in age, gender, or duration of practice. Urinary mercury concentrations were determined on-site with a portable laboratory, using atomic absorption technique methods reported elsewhere. ${ }^{12}$ Samples are analysed within minutes of being collected. The detection limit is $\sim 0.3 \mu \mathrm{g} / \mathrm{l}$. Standards and recalibration of the process are run frequently throughout the assessment period. Mercury concentration results were immediately reported to the dentists, stored on a portable computer, and later entered into an SPSS database for subsequent analysis. Ethanol ingestion was self reported as average number of alcohol units (equivalent of 1 ounce of spirits) per week during the past year. Because certain vitamins may have an antioxidant effect, vitamin usage was surveyed, and included as a covariate in the analyses. Questionnaire data were entered by a data entry service at the University of Washington using double entry techniques to reduce error. Analyses were conducted using SPSS statistical software.

Subjects were initially categorised bivariately as either drinkers or abstainers, as in the above referenced Faroe Island study for analyses utilising the entire subject population. To further investigate the effects of ethanol on urinary $\mathrm{Hg}$ concentration, simple and multivariate linear regression analyses were performed on the total study population, and case-control (logistic regression) analyses were performed on a subset of the study population in which the cases were defined as those individuals with urinary $\mathrm{Hg}$ concentrations of $\geqslant 15 \mu \mathrm{g} / \mathrm{l}(\approx$ top $5 \%, \mathrm{n}=43)$, and controls as those with concentrations of $<1.0 \mu \mathrm{g} / \mathrm{l}(\approx$ bottom $5 \%$, $\mathrm{n}=112$ ).

\section{RESULTS}

Table 1 presents study population characteristics for the study population. Certain personal, professional, and practice characteristics such as number of years in practice, and number of accidental elemental mercury spills in office during the past year, have been shown to significantly influence urinary mercury concentration among dentists, and therefore were included as covariates in linear and logistic regression analyses. ${ }^{13}$ Table 1 also shows study population characteristics for the case and control groups in comparison to the overall study population. The substantially smaller percentage of females in this study $(\sim 8 \%)$ is due to the smaller percentage of females engaged in active dental practice in the USA $(\sim 14 \%)$. Female dentists are additionally underrepresented at professional meetings such at the one at

Table 1 Study population characteristics

All $(n=1171)$ Cases $(n=43)$ Controls $(n=112)$

\begin{tabular}{llcc}
\hline Age, mean (SD) & $49.3(11.1)$ & $53.9(12.8)$ & $48.8(11.2)$ \\
\% female & 7.7 & 4.3 & 7.8 \\
$\begin{array}{l}\text { No. years in practice } \\
\text { No. accidental Hg spills }\end{array}$ & $0.5(2.1)$ & $26.8(13.2)$ & $21.1(11.7)$ \\
in office in past year & $1.28(2.5)$ & $0.1(0.4)$ \\
\% taking vitamins & 41.7 & 37.0 & 42.9 \\
\hline
\end{tabular}

which subjects were recruited, accounting for the slight underrepresentation of females in this study population.

A $t$ test comparing urinary mercury concentrations between abstainers and drinkers in the total sample showed a significant difference in urinary mercury concentrations (two tailed $\mathrm{p}<0.05)$. Drinkers $(\mathrm{n}=826)$ had a mean urinary $\mathrm{Hg}$ concentration of $4.8 \mu \mathrm{g} / \mathrm{l}$ (SD 6.5), while the mean for abstainers $(\mathrm{n}=345)$ was $5.4 \mu \mathrm{g} / \mathrm{l}(\mathrm{SD} 6.8)$.

We examined the ethanol dose per week for a doseresponse relation with urinary $\mathrm{Hg}$ concentration using the questionnaire categories for self reported ethanol dose. Table 2 shows the results. Although the mean $\mathrm{HgU}$ does not exhibit a clear dose-response relation, the median, which exhibits a more clear relation, may provide more meaningful information as there are several outliers in the HgU data, which skews the distribution. Because of the distribution skew, we have used the log of the $\mathrm{HgU}$ for regression analyses in which we investigate the relations of $\mathrm{HgU}$ to ethanol dose.

Simple linear regression of ethanol dose on $\mathrm{HgU}$ provided a model with a significance of $p=0.013$, indicating that ethanol dose is an important predictor of $\mathrm{HgU}$, even without adjustment for potential confounders. A multivariate model which also included the covariates previously shown to influence $\mathrm{HgU}$ in cross sectional studies of dentists, ${ }^{13}$ age, gender, number of accidental $\mathrm{Hg}$ spills in past year, number of amalgams in the dentist's own mouth, number of years in practice, and level of vitamin use provided a model with a significance of $\mathrm{p}<0.001$ for ethanol consumption, adjusted for these potential confounders, indicating that these covariates are additionally important in predicting HgU. In this model, after adjusting for the a priori covariates, the dose of ethanol was inversely related to $\mathrm{HgU}$, with a significance of $\mathrm{p}=0.02$.

Case-control analyses were performed using logistic regression methods to examine the risk of having a urinary mercury concentration in the top of the distribution versus having one in the bottom of the distribution across categories of increasing ethanol consumption. Table 3 shows both the unadjusted and adjusted odds ratios. The reference category was set as that of abstainers, with an odds ratio of 1.0. In both unadjusted and adjusted analyses, a clear protective dose-response relation may be seen, with decreasing risk of being a "case" (having an $\mathrm{HgU} \geqslant 15 \mu \mathrm{g} / \mathrm{l}$ ) with increasing ethanol consumption. Although the relation appears clear in the unadjusted model, it is not statistically significant. The significance of the adjusted model is $p<0.001$, and the $\chi^{2}$ test for trend across ethanol consumption categories in the adjusted model is $\mathrm{p}<0.05$, confirming the dose-response relation.

\section{DISCUSSION}

Prior animal and human studies have indicated that there is an inhibitory effect of ethanol on mercury absorption. To date, the only reported human evidence with a sizeable study population $(\mathrm{n}=1023)$ is that from the Faroe Island study. ${ }^{7}$ This study, although involving a large study population, was

\begin{tabular}{|c|c|c|c|}
\hline $\begin{array}{l}\text { No. alcohol } \\
\text { units/wk }\end{array}$ & $\begin{array}{l}\text { Median } \mathrm{Hg} \text { conc. } \\
(\mu \mathrm{g} / \mathrm{l})\end{array}$ & $\begin{array}{l}\text { Geometric } \\
\text { mean }(\mu \mathrm{g} / \mathrm{l})\end{array}$ & $n$ \\
\hline 0 & 3.8 & 3.45 & 345 \\
\hline $1-3$ & 3.5 & 3.11 & 552 \\
\hline $4-7$ & 3.2 & 2.93 & 130 \\
\hline $8-11$ & 2.8 & 2.69 & 68 \\
\hline$>11$ & 2.8 & 2.71 & 76 \\
\hline
\end{tabular}


Table 3 Unadjusted and adjusted odds ratios

\begin{tabular}{lll}
\hline $\begin{array}{l}\text { No. drinks } \\
\text { alcohol/wk }\end{array}$ & $\begin{array}{l}\text { Odds ratio }(95 \% \mathrm{Cl}) \\
\text { unadjusted }\end{array}$ & $\begin{array}{l}\text { Odds ratio }(95 \% \mathrm{Cl}) \\
\text { adjusted }^{*}\end{array}$ \\
\hline 0 & 1.00 & 1.00 \\
$1-3$ & $0.63(0.29-1.36)$ & $0.72(0.28-1.85)$ \\
$4-7$ & $0.53(0.15-1.87)$ & $0.56(0.13-2.37)$ \\
$8-11$ & $0.38(0.07-1.97)$ & $0.32(0.05-2.2)$ \\
$>11$ & $0.27(0.05-1.31)$ & $0.20(0.04-1.09)$ \\
\hline
\end{tabular}

*Adjusted for age, gender, no. years in practice, no. amalgams in own mouth, no. accidental $\mathrm{Hg}$ spills during past year, and use of vitamins.

only able to provide limited evidence of the relation, reporting that blood mercury levels were slightly lower if the mother had occasionally ingested alcoholic beverages. It should be noted that the primary exposure in this study population was to methyl mercury, which may have contributed to the limited effect detected.

Dentists provide a good study population in which to examine low level mercury exposure. In this case, we were able to obtain information and HgU samples for a large population in a well controlled study situation over a short study period. Although self report was used for many of the variables included in the analyses, the recall period was for only the past year, and the population is a well educated and scientifically motivated one. In our multivariate analyses, even after adjusting for factors known to influence $\mathrm{HgU}$ in dentists, we found clear evidence of the effect of ethanol on $\mathrm{HgU}$. Because certain vitamins are antioxidants, we included the use of vitamins as a covariate, even though there is no good evidence to date that the antioxidant effects of certain vitamins produce an ethanol-like effect on mercury absorption. Although an alternative consideration might be the diuretic effect of ethanol producing a dilution of the $\mathrm{Hg}$ concentration with increasing ethanol dose, the clear inverse dose-response relation seen at even a small number of alcohol units per week (0-7), at levels where a diuretic effect on urinary concentration of $\mathrm{Hg}$ would be insignificant, would indicate that this possibility is not an important factor.

We believe that this straightforward investigation provides the first specific confirmation in a large scale human study of the inhibitory effect of ethanol on urinary mercury concentration, and by inference, on mercury absorption. Further research with human populations may further elucidate this probable antioxidant effect through examining the potential effect of antioxidant vitamin consumption on mercury absorption.

\section{ACKNOWLEDGEMENTS}

This work was partially supported by NIDCR grant DE00415.

\section{Authors' affiliations}

M D Martin, C Naleway, Department of Oral Medicine, University of Washington, Seattle, USA

\section{REFERENCES}

1 Kudsk FN. Absorption of mercury vapor from the respiratory tract in man. Acta Pharmacol Toxicol 1965:23:250-62.

2 Kudsk FN. The influence of ethyl alcohol on the absorption of mercury vapour from the lungs in man. Acta Pharmacol Toxicol 1965;23:263-74.

3 Kudsk FN. Uptake of mercury vapor in blood in vivo and in vitro from $\mathrm{Hg}$ containing air. Acta Pharmacol Toxicol 1969:27:149-60.

4 Hursh JB, Greenwood MR, Clarkson TW, et al. The effect of ethanol on the fate of mercury vapor inhaled by man. Pharmacol Exp Ther 1980;214:520-7.

5 Dunn JD, Clarkson TW, Magos L. Interaction of ethanol and inorganic mercury: generation of mercury vapor in vivo. J Pharmacol Exp Ther $1981 ; 216: 19-23$

6 Langworth S, Elinder CG, Göthe CJ, et al. Biological monitoring of environmental and occupational exposure to mercury. Int Arch Occup Environ Health 1991:63:161-7.

7 Grandjean P, Weihe P, Jorgensen PJ, et al. Impact of maternal seafood diet on fetal exposure to mercury, selenium and lead. Arch Environ Health 1992;47:185-95.

8 Sallsten G, Kreku S, Unosson H. A small dose of ethanol increases the exhalation of mercury in low-level exposed humans. J Toxicol Environ Health A 2000;60:89-100

9 Magos L, Clarkson TW, Greenwood MR. The depression of pulmonary retention of mercury vapor by ethanol: identification of the site of action. Toxicol Appl Pharmacol 1973;26:180-3.

10 Martin MD, McCann T, Naleway C, et al. The validity of spot urine samples for low-level occupational mercury exposure assessment and relationship to porphyrin and creatinine excretion rates. J Pharmacol Exp Ther 1996:277:239-44.

11 Woods JS, Martin MD, Leroux BG. Validity of spot urine samples as a surrogate measure of 24-hour pophyrin excretion rates. J Occup Environ Med 1998:40:1090-101.

12 Naleway C, Chou H-N, Muller T, et al. On-site screening for urinary Hg concentrations and correlation with glomerular and renal fubular function. $J$ Public Health Dent 1991;51:12-17.

13 Martin MD, Naleway C, Chou H-N. Factors contributing to mercury exposure in dentists. JADA 1995;126:1502-11. 